\title{
Gypsy endogenous retrovirus maintains potential infectivity in several species of Drosophilids Jose V Llorens ${ }^{1}$, Jonathan B Clark ${ }^{2}$, Isabel Martínez-Garay ${ }^{1}$, Sirena Soriano ${ }^{1}$, Rosa de Frutos ${ }^{1}$ and María J Martínez-Sebastián*1
}

\author{
Address: ${ }^{1}$ Departament de Genètica, Universitat de València, 46100-Burjassot, Valencia, Spain and ${ }^{2}$ Department of Zoology, Weber State \\ University, Ogden, 84408-2505 Utah, USA \\ Email: Jose V Llorens - jovillo@uv.es; Jonathan B Clark - jclark1@weber.edu; Isabel Martínez-Garay - imgaray@uv.es; \\ Sirena Soriano - sirena.soriano@uv.es; Rosa de Frutos - rosa.frutos@uv.es; María J Martínez-Sebastián* - maria.jose.martinez@uv.es \\ * Corresponding author
}

Published: 31 October 2008

BMC Evolutionary Biology 2008, 8:302 doi:10.1 |86/I47|-2|48-8-302

This article is available from: http://www.biomedcentral.com/I47I-2/48/8/302

(c) 2008 Llorens et al; licensee BioMed Central Ltd.

This is an Open Access article distributed under the terms of the Creative Commons Attribution License (http://creativecommons.org/licenses/by/2.0), which permits unrestricted use, distribution, and reproduction in any medium, provided the original work is properly cited.
Received: 12 June 2008

Accepted: 31 October 2008

\begin{abstract}
Background: Sequences homologous to the gypsy retroelement from Drosophila melanogaster are widely distributed among drosophilids. The structure of gypsy includes an open reading frame resembling the retroviral gene env, which is responsible for the infectious properties of retroviruses.
\end{abstract}

Results: In this study we report molecular and phylogeny analysis of the complete env gene from ten species of the obscura group of the genus Drosophila and one species from the genus Scaptomyza.

Conclusion: The results indicate that in most cases env sequences could produce a functional Env protein and therefore maintain the infectious capability of gypsy in these species.

\section{Background}

Gypsy (gypsyDm) is an endogenous retrovirus of D. melanogaster. Its structure is largely similar to the vertebrate retroviruses and it possesses infective properties [1-4]. Sequences homologous to gypsyDm are widely distributed among Drosophila species, found in both the subgenus Sophophora as well as in the subgenus Drosophila [5-9]. In addition, they have been detected in some species of the genus Scaptomyza $[8,9]$. The phylogenetic relationships among gypsy sequences from Drosophila species is not be always coincident with those of their hosts, which could be indicative of horizontal transfer during evolutionary history $[6,7,9,10]$. Horizontal transfer has been invoked to explain the evolutionary patterns of several families of transposable elements [11-15]. In some cases, such as the P element, the horizontal transfer events are strongly doc- umented [16-18]. However, the underlying mechanisms to horizontal transfer remain elusive.

In the case of gypsyDm, the horizontal transfer could be associated with the infectious properties of that element. One way to evaluate the potential impact of the gypsy infectious ability on the evolutionary history of gypsy sequences from Drosophila, is to analyze the infective ability of gypsy homologous sequences from species other than D. melanogaster.

The infectious ability of $g y p s y D m$ is associated with the expression of a retroviral envelope-like protein encoded by the env gene [2]. Structural analysis of the env region of a given gypsy sequence is the first step in determining its potential infectious ability. In previous reports, two com- 
plete gypsy elements from $D$. subosbcura and D. virilis were sequenced $[10,19]$. Although, the env region is preserved in its full-length, in both species single indels result in potentially truncated Env proteins [10]. However, as has been proposed in [4], it is possible that both, defective and complete fully functional gypsy elements, coexist in the genome of these species. This study describes the existence of full-length $e n v$ genes in the genomes of D. subosbcura and D. virilis, as well as in the genomes of the several species closely related to D. melanogaster:D. simulans, $D$. erecta, D. orena, D. teissieri, and D. yakuba. Interestingly, these gyspy sequences can potentially invade the D. melanogaster genome, and escape the control normally exerted by the flamenco gene, whose product represses mobility[4].

It is generally accepted that species of the obscura group can be classified into five subgroups: the pseudobscura and affinis subgroups, consisting of Neartic species, the subobscura and obscura subgroups, mainly consisting of Paleartic species, and the microlabis subgroup encompassing Afrotropical species [20-22]. Gypsy sequences homologous to gypsyDm have been detected in several species of the Neartic and Paleartic subgroups, appearing as a monophyletic group that is highly diverged from the gypsyDm prototypic element [7,8]. With the exception of $D$. guanche, the 5' region of the env gene in all species examined could potentially produce a functional N-terminal region of the Env protein. To confirm the existence of potentially infective gypsy lineages other than gypsyDm, sypsyDs, and gypsyDv, we report here an analysis of the complete env gene of ten species of the obscura group and one species from the genus Scaptomyza. Most of the species analyzed contain intact copies of the env region and the env splicing sites, and in vitro can be translated into a protein of the predicted size.

\section{Results and discussion}

Sequences homologous to gypsy from D. melanogaster are widely distributed among Drosophila species. In previous studies, the complete gypsy elements from D. subobscura and D. virilis have been sequenced [10]. These studies were carried out to understand the evolutionary behaviour of gypsy and indicate that the genetic organization of gypsy in those species is conserved. Moreover, in D. melanogaster do the gypsy elements have a complete functional env gene [10]. In the case of D. virilis and D. subobscura, it has been shown that the genomes of these species contain at least one copy of gypsy putatively encoding a complete envelope protein [4].

The main aim of this study is to examine whether potentially functional copies of the env region of gypsy exist in other species. This was accomplished by searching genomes for the presence of full-length env coding sequences and assessing their functionality.

\section{The analysis of the env sequences shows their infectious capability}

To analyze the infectious capability of the gypsy element of each analyzed species (table 1) we amplified the env region with specific primers designed from the intergenic sequence (figure 1 ). In all species the size of the amplified sequence was $1.5 \mathrm{~kb}$, except for $D$. algonquin and $D$. tolteca, with sizes of 0.5 and $1.2 \mathrm{~kb}$ respectively. The PCR products of all species, were cloned in $\mathrm{PCR}^{\circledR} 2.1-\mathrm{TOPO}^{\circledR}$ (Invitrogen) and sequenced with the specific primers of the vector, demonstrating that $D$. algonquin and $D$. tolteca had a deletion of $1.5 \mathrm{~kb}$ and $0.3 \mathrm{~kb}$ respectively. In the same way, D. pseudoobscura and D. miranda had deletions of 40 $\mathrm{pb}$ and $21 \mathrm{pb}$ respectively that produced a stop codon and an Env truncated protein. D. persimilis had a single nucleotide change, also producing a stop codon. In contrast, $D$. madeirensis, D. obscura, D. ambigua, D. bifasciata, D. affinis and $S$. elmoi had different polymorphisms but none resulting in a truncated protein, so these env regions would produce potentially active proteins.

With these data, we can conclude that D. madeirensis, D. obscura, D. ambigua, D. bifasciata, D. affinis and S. elmoi

Table I: List of species used in the analysis of env sequences.

\begin{tabular}{|c|c|c|c|c|}
\hline Genus & Subgenus & Group & Subgroup & Species \\
\hline \multirow[t]{10}{*}{ Drosophila } & Sophophora & obscura & subobscura & D. madeirensis \\
\hline & & & obscura & D. obscura \\
\hline & & & & D. ambigua \\
\hline & & & & D. bifasciata \\
\hline & & & pseudoobscura & D. pseudoobscura \\
\hline & & & & D. persimilis \\
\hline & & & & D. miranda \\
\hline & & & affinis & D. affinis \\
\hline & & & & D. algonquin \\
\hline & & & & D. tolteca \\
\hline Scaptomyza & Parascaptomyza & & & S. elmoi \\
\hline
\end{tabular}




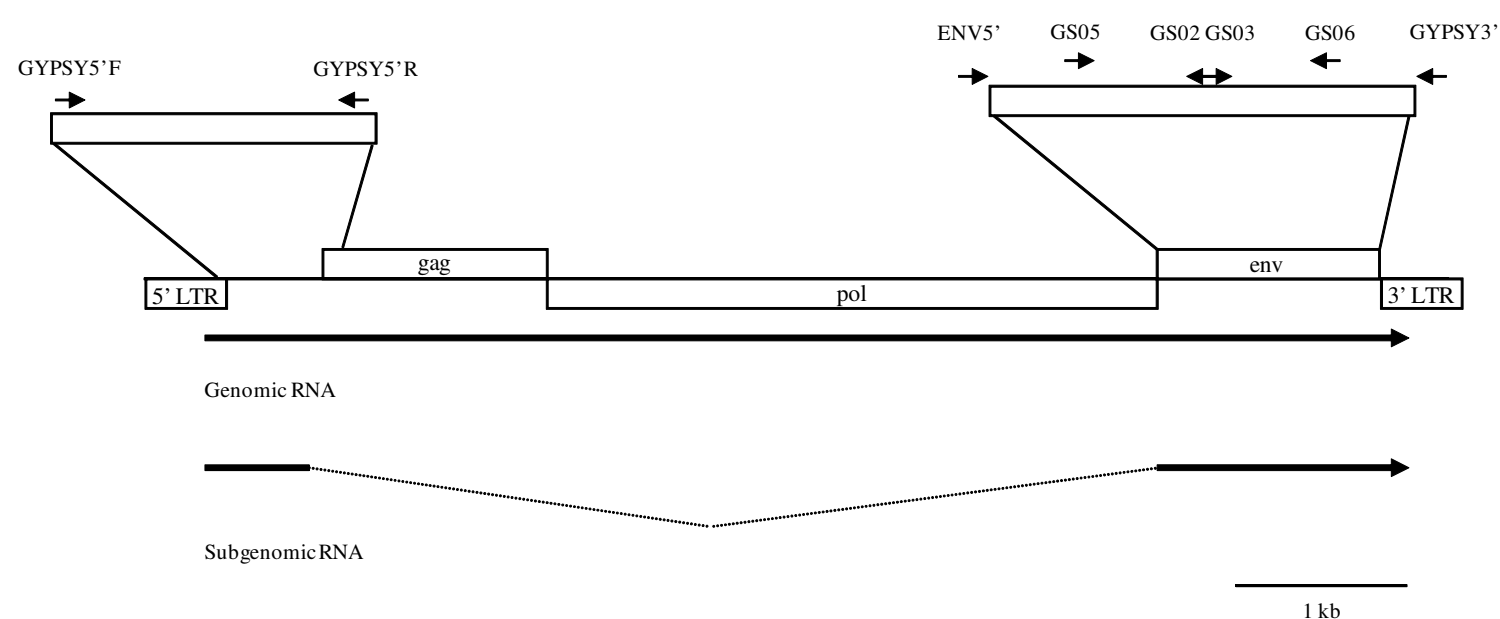

Figure I

Schematic diagram of the gypsy element. The organization of the gypsy element and the genomic and subgenomic RNAs is shown along with the localization of the primers used in this study.

could produce a complete Env protein and therefore could maintain the infectious capability of gypsy in these species.

The env sequences obtained in different species have the capacity to produce complete envelope proteins

The RTS system was used to scan for premature termination mutations and analyze the coding capacity of the gypsy env gene present in the genome of the different species studied. We used genomic DNA from each species to amplify the env sequence. We cloned the obtained sequences into $\mathrm{pCR}^{\circledR} 2.1$-TOPO (Invitrogen) and then we used these clones to produce the PCR products for in vitro protein expression (see material and methods). a

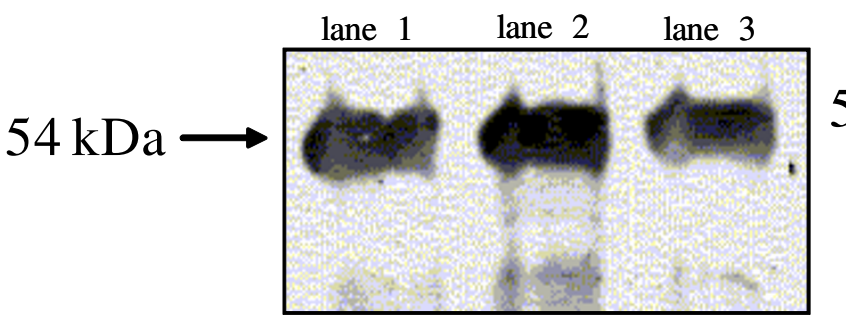

C

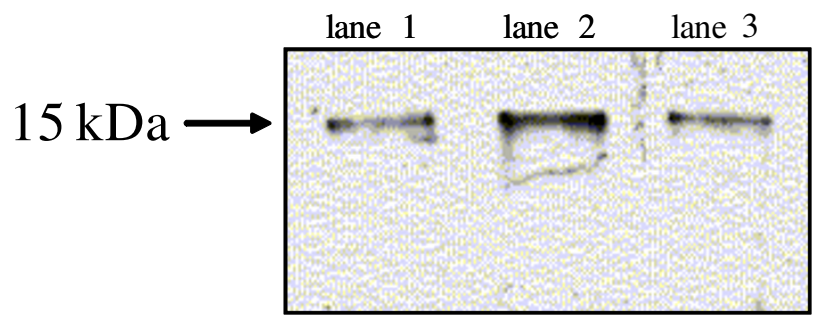

$\mathrm{b}$ $54 \mathrm{kDa} \rightarrow \longrightarrow-$

\section{Figure 2}

In vitro protein sintesis using the RTS system. a) and b) The proteins obtained, using complete sequences of env as templates had the sizes of normal Env protein (54 kDa). a) lane I, D. madeirensis; lane2, D. obscura; lane3, D. ambigua; and b) lane I, D. bifasciata; lane2, D. affinis; lane3, S. elmoi. c) The proteins obtained using disrupted sequences of env as templates were truncated (I5 kDa). c) lane I, D. pseudoobscura; lane2, D. persimilis; lane3, D. miranda. 
The major protein products obtained in this assay have molecular weights corresponding to the expected size for Env protein of D. melanogaster, about $54 \mathrm{kDa}$. These protein products were obtained in the same species where the env nucleotide sequence was complete, without disruptions. We obtained complete Env protein from D. madeirensis, D. obscura, D. ambigua, D. bifasciata, D. affinis and $S$. elmoi, while we obtained truncated proteins from the species where the sequence was disrupted, D. pseudoobscura, D. persimilis and D. miranda (figure 2).

In D. melanogaster, the splicing produces a start codon using AT from 5' site in the gag region and a G from the 3' site in the env region [2]. We have analyzed the sequences from the different species of this study and only D. madeirensis, D. obscura, D. ambigua, D. bifasciata, D. affinis and $S$. elmoi, have conserved the sequences that produce the start codon after the splicing.

These results show that these species contain at least one copy of gypsy element that can encode an envelope protein. Other data that support the existence of functional Env proteins include the presence of N-glycosylation sites, conserved cysteine residues and endopeptidase cleavage sites in all the species with a complete env sequence and the ATG codon (figure 3), as described in $[2,4]$. Moreover, those species have the sequence pattern (R-X(2)-R-X $(5,6)$ [GE]-X(5)- [LV]-X-G-X(2)-D-X(2)-D) for the detection in silico of endogenous retroviral envelope protein proposed in [23]. Only the signal peptide and the membrane-spanning domain sequence show differences on comparing to D. melanogaster gypsy sequence.

\section{Although sequences have the capability to produce complete envelope protein, their expression could be repressed in the species studied}

To demonstrate the capability of the gypsy elements to produce an active envelope protein, we wanted to know if the env gene was expressed in vivo in the species with the complete env sequence.

In order to detect the production of the env mRNA we performed a RT-PCR analysis using the GS05 and GS06 primers with the mRNA extracted from ovarian chambers of the female flies. Since we also wanted to detect alternative splicing, necessary to produce ENV, we used the primers GYPSY5'F and GS06 of the env gene using the same mRNA as before (see materials and methods).

Amplification with the internal primers was successful in all species analysed as well as in the positive control (data not shown). However, alternative splicing was not detected (data not shown). These results suggest that the alternative splicing of gypsy in the obscura species group and $S$. elmoi would be repressed by a genomic gene as occurs in D. melanogaster with the flamenco gene [24]. Alternatively, the gypsy element may use an alternative start codon to express the env region or we simply could not detect the alternative splicing.

\section{The gypsy phylogeny is not totally consistent with species phylogeny}

Since gypsy elements can potentially jump from one individual to another without the need of a vector, it has been proposed that horizontal transfers of gypsy elements is favoured by their ability to encoded full-length ENV proteins [4]. In this way we performed a phylogeny analysis of the env gypsy sequences studied, looking for evidences of horizontal transfer events.

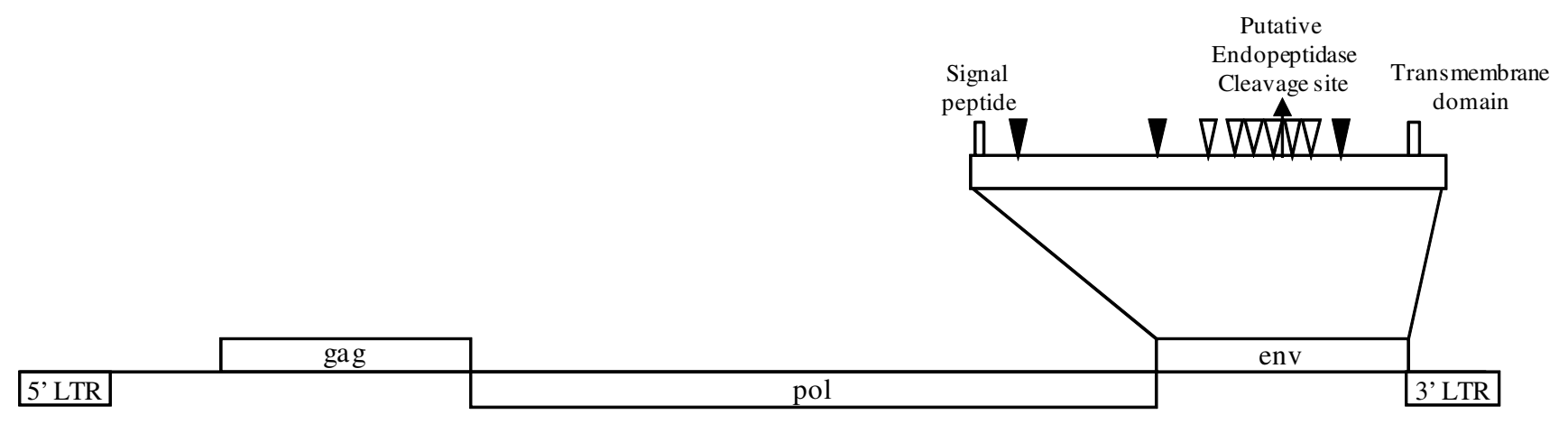

\section{Figure 3}

Schematic structure of the functional env gene in the species analysed. The location of the signal peptide of the precursor protein, the transmembrane domain, and the cellular endopeptidase cleavage site is indicated. The three asparagine ( $\mathrm{N}$ ) residues near the putative glycosylation sites are shown with filled arrowheads. The six cysteine $(\mathrm{C})$ residues are denoted by open arrowheads. 
A Neighbor-joining phylogenetic tree of envelope sequences is shown in figure 4 . The obscura sequences fall into four main clades that are generally consistent with species phylogeny. Clade $A$ includes sequences from $D$. affinis of the affinis subgroup; clade B, D. ambigua, D. bifasciata, D. madeirensis, and D. obscura of the obscura subgroup; clade C, D. miranda, D. persimilis, and D. pseudoobscura of the pseudoobscura subgroup; and clade D, $D$. tolteca of the affinis subgroup. The D. virilis sequence is responsible for the relatively low support for clade A; removing the $D$. virilis sequence from the analysis increases the bootstrap values to $98 / 77$ for a grouping of the $D$. hydei sequence with those from $D$. affinis, with the rest of the topology unchanged. With the exception of the placement of sequences from S. elmoi, the topology of this tree is identical to that obtained by parsimony analysis, which yielded a shortest tree of 1477 steps (Consistency Index $=0.8$; Retention Index $=0.9$ ). The distribution of sequence from $D$. affinis and $D$. tolteca into two distinct clades is an unexpected result that is consistently seen in all analyses. Constraining the analysis so that the sequences from D. tolteca and D. affinis are monophyletic adds an additional nine steps to the parsimony tree.

The potential effect of recombination on the phylogeny was assessed by using the GARD algorithm, which uses maximum likelihood models to detect recombination events. The BIC model found no evidence of recombination, while the AIC model identifies a potential breakpoint at position 1288 in the 1392 character alignment. The potential effect of this event on the phylogeny was also examined using a sliding-window approach. Separate phylogenetic analyses were constructed from four 400-bp regions of the envelop alignments (positions 1-399; 400799; 800-1199; and 1200-1493), and on a fifth dataset that excluded the region beyond the breakpoint. The latter analysis resulted in an identical tree to that shown in figure 4 , as did two of the four sliding window analyses. In the other two sliding-window trees, the $D$. virilis sequence was excluded from clade $\mathrm{A}$, as it was in one of two alternate trees obtained from the AIC analyses. Regardless of its precise affiliation, in an expanded phylogenetic analysis that includes 180 published gypsy sequences, D. virilis clearly belongs to a well-supported clade that includes all of the obscura sequences (see below).

Sequences from S. elmoi fall into clade B, in which they form two subclades. Although not monophyletic, the average amount of sequence divergence between the two subclades is just $1.1 \%$, and if the sequences from $S$. elmoi are constrained to be monophyletic the total length of the parsimony tree is unchanged. If the gypsy sequences from S. elmoi are excluded from the analysis, the topology of the obsucra sequences is unchanged from that shown in figure 4. To make the gypsy phylogeny conform completely to the species phylogeny (including S. elmoi as an outgroup, a monophyletic clade that includes $D$. hydei and D. virilis, and D. melanogaster as a sister taxon to the obscura sequences) increases the parsimony tree length from 1477 to 1542 steps. Thus, there is clear incongruence between the gypsy phylogeny and that of the species from which the sequences were isolated.

The envelope sequences from $S$. elmoi differ on average by only $2.1 \%$ from those found in the obscura species of clade B. As a hallmark of horizontal transfer is the existence of highly similar sequences in the genomes of divergent taxa [15], transfer of gypsy from the obscura group to the genus Scaptomyza seems likely. Because the sampling from $S$. elmoi is quite extensive, an explanation of the retention of an ancestral polymorphism for the phylogenetic incongruence seems unlikely. This conclusion is further supported by comparison to the divergence of a non-mobile nuclear gene, Adh. Over exon 2 of this gene, the average divergence between $S$. elmoi and the clade B Adh sequences is $20.0 \%$. A horizontal transfer involving the genus Scaptomyza and the Palearctic members of the obscura species group is of particular interest because of the well-documented horizontal transfer of the P transposable element between these taxa [17]. In that case, the direction of transfer was almost certainly from Scaptomyza to D. bifasciata. In the case of gypsy, the phylogeny indicates a transfer in the opposite direction.

To provide some comparison to other studies of gypsy evolution, an extensive phylogenetic analysis was performed with an enlarged dataset of 180 sequences, combining those from this study with those of [9] and [26]. This allows us to assess the coherence of the two clades, A and $\mathrm{B}$, which include postulated horizontal transfer events, and to increase the sample size considerably to examine the alternative hypothesis of ancient lineages. This tree is presented in figure 5 . The positions of the D. hydei, D. virilis and $S$. elmoi sequences remain unchanged from that shown in figure 4, providing additional evidence to support the hypothesis of horizontal transfer.

\section{Molecular evolutionary analysis of gypsy sequences is consistent with activity}

To examine the evidence for selection on the gypsy sequences, the numbers of synonymous substitutions $(d S)$ and nonsynonymous substitutions $(d \mathrm{~N})$ were calculated for each sequence. The average $d \mathrm{~N} / d \mathrm{~S}$ ratio was then calculated for each clade (see table 2), providing some indication of whether purifying selection is or has been operating on these sequences. For all major clades, the $d \mathrm{~N} / d \mathrm{~S}$ ratio is similar to that obtained for non-mobile nuclear genes [25]. Similar results have been obtained in a number of studies of gypsy sequence evolution [6,8-10]. Together, these comparisons suggest that many gypsy ele- 


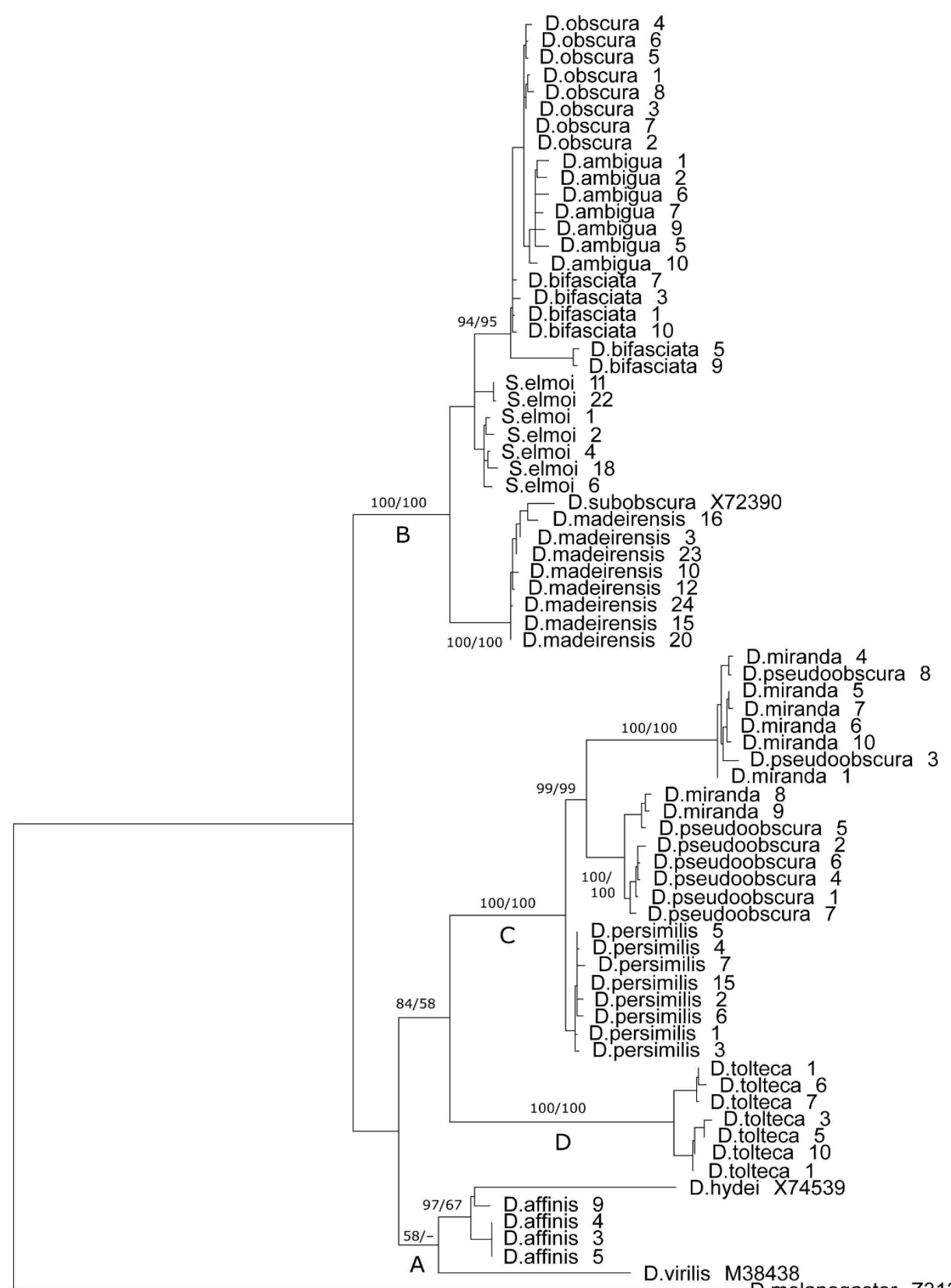

-0.005 substitutions/site

Figure 4

Neighbor-Joining phylogeny of gypsy sequences from the obscura species group. The four main clades, discussed in the text, are identified by letters. Numbers on branches represent bootstrap percentages from 1000 replicates as determined by Neighbor-Joining (value before slash) and parsimony (after slash). Values are shown only for the major clades. The scale bar denotes branch lengths in substitutions per site. 


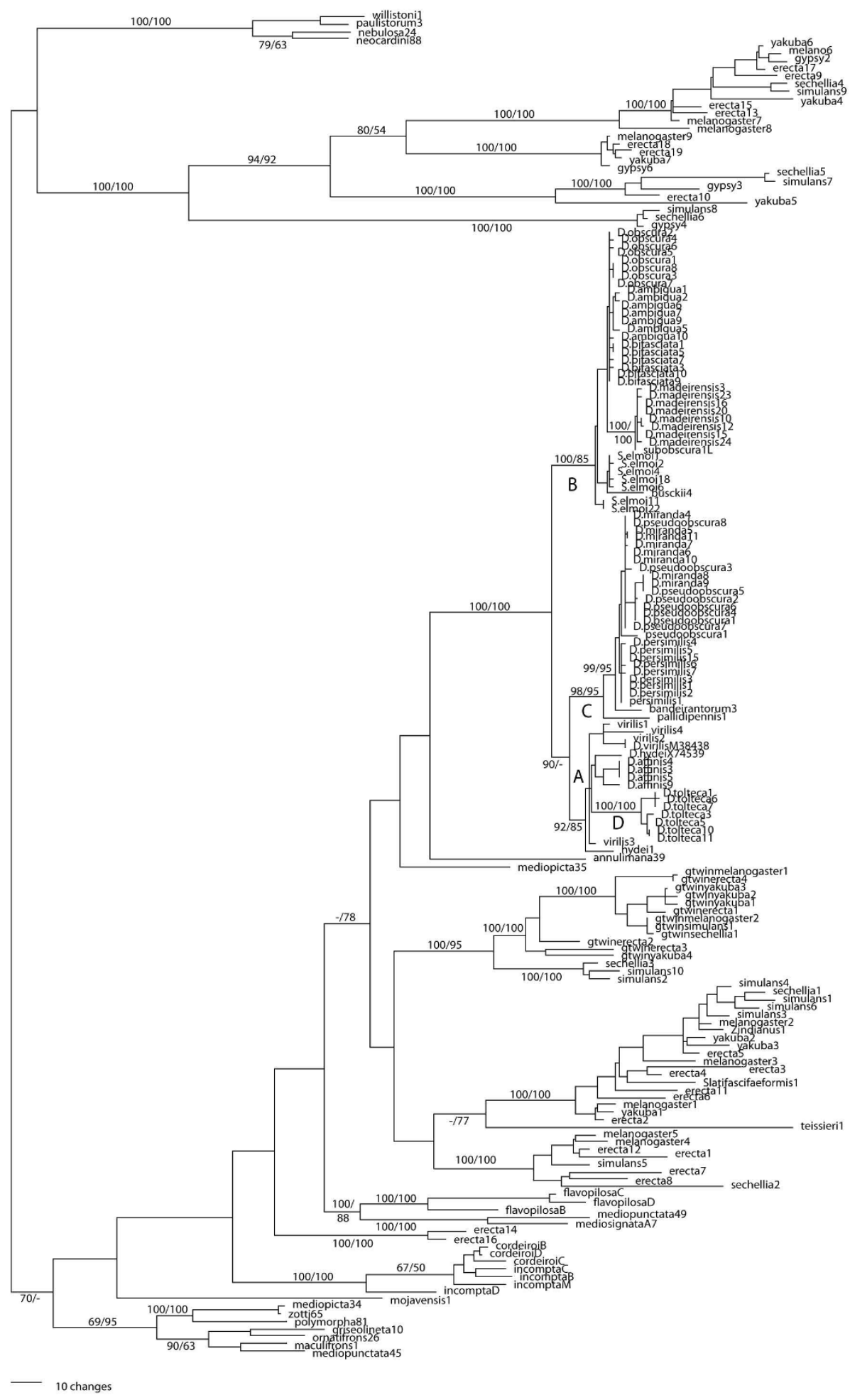

Figure 5

Neighbor-joining phylogenetic tree of 180 envelope sequences. Taxon names preceded by a "D." (obscura species group) or "S." (Scaptomyza elmoi) are from this study. All other sequences are from GenBank, [9] and [26]. Bootstrap values are shown for distance (before slash) and parsimony (after slash) analyses. Values are shown for major clades only. The four clades associated with this study are labeled $A, B, C$, and D, as in figure 4. 
Table 2: The ratio of nonsynonymous to synonymous substitutions for the envelope-coding region of the gypsy element.

\begin{tabular}{cc}
\hline Clade & dN/dS Ratio \\
\hline A & 0.13 \\
B & 0.14 \\
C & 0.16 \\
D & 0.28 \\
\hline
\end{tabular}

Calculations were based on the model of Nei and Gojobori (1986) and include obscura sequences only. Clade designations are given in figure 4.

ments are capable of activity, consistent with the expression data described above. Although the pattern is consistent with activity (and potential infectivity), this conclusion may be complicated by two factors: (i) codon bias, which is lower for the gypsy sequences, when compared to $A d h$ (data not shown), and (ii) the nature of selection on the gypsy sequences which may include functional constraints on the RNA intermediate in addition to selection for a functional envelop protein.

It is noteworthy that clade B includes the gypsy sequences that have intact envelope genes (D. ambigua, D. bifasciata, D. madeirensis, D. obscura, and S. elmoi), whereas clades D and $\mathrm{C}$ consist of those with interrupted reading frames. Clade A comprises sequences from the Neartic species $D$. affinis, which also encode full-length envelope proteins and are characterized by very low $d \mathrm{~N} / d \mathrm{~S}$ ratios. It is fascinating that in the two clades with open envelope reading frames and low $d \mathrm{~N} / d \mathrm{~S}$ ratios, horizontal transfer has likely occurred. Even though the gypsy sequences examined from clade $\mathrm{C}$ are not likely functional, the low $d \mathrm{~N} / d \mathrm{~S}$ ratio suggests that these elements have been active in the recent past. The envelope coding regions of D. tolteca (clade D) are characterized by a substantial deletion at the beginning of the envelope gene and relatively higher $d \mathrm{~N} / d \mathrm{~S}$ ratios, suggesting that they have been inactive for considerably longer than the clade $\mathrm{C}$ elements. Thus, the phylogenetic and sequence analyses together provide a snapshot of the evolutionary history of gypsy in the obscura species group that is consistent with their activity and potential infectivity.

Horizontal transfer of gypsy has been suggested in other studies: among sequences from the obscura group and $D$. virilis/D. hydei, which belong to a different subgenus [7], and within the melanogaster species group [6]. Two additional studies, which are similar is scope to the one described here, concluded that horizontal transfer of gypsy was fairly widespread among species in the genus Drosophila and sister genera $[9,26]$. As these studies included a total of only three sequences from the obscura group, they were not able to identify precisely the species involved in the transfer events. In the analysis presented here, the envelope sequence from $D$. hydei shows a strong affiliation to the sequences from $D$. affinis; a less well-supported clade includes the sequences from $D$. virilis as well. As the envelope sequences from $D$. hydei, D. virilis, and $D$. affinis differ by an average of only $6.2 \%$, and the species sampling is now quite extensive (see figure 5), horizontal transfer remains the best explanation for the incongruent phylogeny. The hypothesis of horizontal transfer finds additional support in a comparison of the divergence of the envelop region to the non-mobile gene, $A d h$. The average divergence of $A d h$ sequences from $D$. hydei/D. virilis and D. affinis is $21.4 \%$ (clade A).

Using the values for synonymous nucleotide substitutions, [26] provided an estimate of 6.3 million years ago (MYA) for a transfer from the obscura group (as represented by $D$. persimilis) to $D$. virilis. When a similar analysis is done with the $D$. affinis sequences (which are not included in [26]), an estimate of only 2.7 MYA is obtained for a transfer from $D$. affinis to D. hydei, and 3.1 MYA for a transfer from $D$. affinis to $D$. virilis. In clade $\mathrm{B}$, time estimates calculated from synonymous changes place the transfer from the Paleartic obscura to S. elmoi at 2.1 MYA. The fact that these transfers occurred relatively recently supports the idea that at least some genomes in the obscura group harbor active, infectious gypsy elements.

\section{Conclusion}

The genomes of six of the eleven species of analysed encode intact copies of the envelop coding region of gypsy and retain intact envelop splice sites. Their potential for expression was analyzed in vitro and each was found to encode a protein whose size is consistent with activity. Moreover, these elements possess two additional features of active gypsy genes, alternative splice sites and the use of alternative start codons. Evolutionary analysis of the envelop sequences shows that interspecies transfer of $g y p s y$ is associated with those obscura elements that can encode a functional envelop protein. Such horizontal transfer is supported by the incongruence of the gypsy and species phylogenies and by the low levels of gypsy divergence when compared to the divergence of host genes from the same species.

\section{Methods}

\section{Drosophila and Scaptomyza stocks}

Ten species from the obscura group (D. madeirensis, D. obscura, D. ambigua, D. bifasciata, D. pseudoobscura, D. persimilis, D. miranda, D. affinis, D. algonquin, D. tolteca), and one species from the Scaptomyza genus (S. elmoi) were analysed. All species were obtained from the Tucson Stock Center (table 1). 


\section{PCR amplification of the env coding region}

Genomic DNA from each species was prepared as in [7]. PCR was usually carried out using 100 ng of genomic DNA, 10 pmol of each primer, $10 \mathrm{mM}$ dNTPs and $0.5 \mathrm{U}$ of Netenzyme DNA polymerase. The amplifications were performed in a Eppendorf Mastercycler 5333 version 2.01.33-09. The amplified 1443-bp fragment contains a short intergenic stretch, and the entire ORF3 region from the gypsy element. The pairs of primers used to amplify the $e n v$ region in the differents species were (see figure 1): ENV5'mad: 5'-AGTAGAGTTAGAATAACGTCC-3' and GYPSY3': 5'-TRGCGMGTCAGCATTGTT-3' (where $\mathrm{R}$ is $\mathrm{G}$ or A) for D. madeirensis, D. ambigua, D. bifasciata and $S$. elmoi; ENV5'obs: 5'-ATTAGAGTTAGAACAACGT CT-3' and GYPSY3' (see sequence above) for D. obscura; ENV5'pseuaff: 5'-CTTAGAGTTAGAACACCGTCT-3' and GYPSY3' for D. pseudoobscura, D. persimilis, D. affinis, D. algonquin; ENV5'mir: 5'- CTTAGAGTTAGAACACCAT CT3' and GYPSY3' for D. miranda; ENV5'tol: 5'- CTTAGAGTTAGAACACCTTCT-3' and GYPSY3' for D. tolteca.

PCR conditions consisted of a single denaturing step at $94^{\circ} \mathrm{C}$ for $5 \mathrm{~min}$, followed by 30 cycles at $94^{\circ} \mathrm{C}$ for $30 \mathrm{~s}$, $55-60^{\circ} \mathrm{C}$ for $1 \mathrm{~min}$ (depending on each primer), and $72^{\circ} \mathrm{C}$ for $1 \mathrm{~min} 30 \mathrm{~s}$; a final extension was performed at $72^{\circ} \mathrm{C}$ for $10 \mathrm{~min}$.

To amplify the 5' region of gypsy, which contains the env splicing site, different primers, derived from alignments of sypsy elements from D. subobscura, D. virilis and D. melanogaster were designed. The size of the amplified fragment is $500 \mathrm{bp}$. The sequences of these primers were (see figure 1): GYPSY5'F: 5'-GCKWTGATGGCGTATGCATTG-3' (where $\mathrm{K}$ is $\mathrm{G}$ or $\mathrm{T}$ and $\mathrm{W}$ is $\mathrm{A}$ or $\mathrm{T}$ ) and GYPSY5'R: $5^{\prime}-$ YTATGCTGCCGAAAGTATGC-3' (where Y is C or T).

\section{Cloning and DNA sequencing}

PCR products were cloned into $\mathrm{PCR}^{\circledR} 2.1-\mathrm{TOPO}^{\circledR}$ (Invitrogen). Eight clones from each species were sequenced in an automatic ABI-Prism sequencer. The multiple alignments of gypsy ORF3 sequences were performed with the program SEQUENCHER version 4.0.5 (Gene Codes Corporation). The sequencing reactions were carried out with the primers (see figure 1): ENV5' and GYPSY3' (see sequence above) GS05, 5'-TAA TAC TCA CGA TAA CGT TGG-3' for D. bifasciata, D. madeirensis and D. ambigua, GS04, 5'-TAA TAC TCA CCA TAA CGT TGG-3' for D. affinis and GSO6, 5'-ATG TCC GAT GAT GTT TAG GAG-3', GSO2, 5'-CACTTAAATTCAACTTTGGGG-3' and GS03, 5'-CCAAAGTTGAATTTAAGTGCC-3' for all species.

All sequences obtained in this study were submitted to the EMBL Nucleotide Sequence Database [EMBL: $\underline{\text { AM748829 }}$ - $\underline{\text { AM748898]. }}$

\section{Phylogenetic and sequence analysis}

Nucleotide sequences were aligned using the default settings of ClustalW http://www.ebi.ac.uk/clustalw/ and were then refined by eye to correct obvious misalignments. The phylogeny of 64 gypsy sequences from the obsura group and seven sequences from Scaptomyza elmoi was examined using parsimony, distance, and maximum likelihood methods as implemented by PAUP* $4.0 \mathrm{~b} 10$. Four additional gypsy sequences, from D. subobscura [X72390], D. hydei [X74539], D. virilis [M38438], and D. melanogaster [Z31368] were also included in the analysis. A total of 1392 positions were analysed, with gaps treated as missing data. Identical tree topologies were obtained for all major clades using each method, and adjusting the search parameters for each. The Neighbor-Joining tree shown in figure 4 was constructed using the Kimura twoparameter model for nucleotide substitution. The parsimony analyses, which included 553 informative characters, used random addition of sequences (10 replicates) and TBR branch swapping; both heuristic and branchand-bound searches yielded identical trees. The maximum-likelihood analyses used empirical base frequencies and a substitution model in which all rates were equal.

Previous studies of gypsy phylogeny indicate that the gypsy sequence from $D$. melanogaster is an appropriate outgroup for phyolgenetic analysis of sequences from the obscura group (e.g., [26]). The use of this taxon as an outgroup was verified in two additional ways. First, it is clearly the most divergent of the gypsy sequences, showing an average distance of $36 \%$ (Kimura two-parameter method) from the other sequences. Second, midpoint rooting with both neighbor-joining and parsimony establish the root along the D. melanogaster branch. Bootstrap values were determined from 1000 replicates for both parsimony and Neighbor-Joining. Character state analyses and parsimony tree-length comparisons were determined with MacClade 4.0.3. The potential effect of recombination on the phylogenetic analyses was assessed with the GARD method [27].

Molecular evolutionary analysis was implemented with MEGA4 [28]. Nucleotide divergences were calculated using the Kimura two-parameter method and the numbers of synonymous substitutions (dS) and nonsynonymous substitutions $(d N)$ were calculated using the Nei and Gojobori (1986) [29] codon model for the nucleotide sequences that had been aligned based on amino acid position. To facilitate comparisons, the alignments were adjusted by eye to maintain homologous codon positions. Because of some variability in identifying the start site in all sequences, the comparison began at a conserved serine codon, which, in most sequences, is the eighth amino acid of the envelope polypeptide. The divergence of gypsy sequences was compared to those of a non-mobile 
nuclear gene, Adh. Sequences for D. hydei, D. virilis, $S$. elmoi, and all available taxa of the obscura species group were obtained from GenBank. The comparisons were limited to exon 2, the only sequence available for some species.

\section{In vitro translation of the Env proteins}

To generate PCR products for in vitro protein expression, special primers, consisting of two different parts, were designed. The first half adds regulatory elements necessary for expression in a prokaryotic system, such as a T7 promoter, the ribosomal binding site and the $\mathrm{T} 7$ terminator. The second half recognizes a specific region of env, which corresponds to the sequence used to amplify the env region in the different species (see sequences above). This PCR product can be expressed directly in E. coli HY using the Rapid Translation System (RTS) from Roche. The E. coli Linear Template Generation Set, His-tag and the RTS 100 E. coli HY (both purchased from Roche), were used to detect mutations that lead to the termination of mRNA translation and subsequently to protein truncation. The translation products were separated by discontinuous SDS-PAGE through a $12 \%$ separating gel with Tris-glycine buffer. The signals were detected by colourimetric system.

\section{RT-PCR env amplification}

RNA extractions were carried out using approximately 100 mg of flies. These RNA extractions were carried out with the QuickPrep ${ }^{\mathrm{TM}}$ Micro mRNA Purification Kit (Amersham Biosciences), according to the manufacturer's instructions. Afterwards, the mRNA was treated with RQ1 RNasefree DNase (Promega) to remove remainder DNA of the sample. RNA obtained was reverse transcribed in the presence of AMV-RT (Promega) and the env-specific primer GS06 (see sequences above).

Reactions were carried out at $37^{\circ} \mathrm{C}$ for 2 hours followed by 10 minute at $65^{\circ} \mathrm{C}$ to denature the enzyme. From the first strand reaction, $2 \mu \mathrm{l}$ was used as a template in PCR using the following primers: GS05, for D. bifasciata, D. madeirensis and D. ambigua, GS04, for D. affinis and GSO6 (see sequences above) for all species (figure 1).

To analyse the alternative splicing product, the RNA obtained was reverse transcribed and amplified with GS05 and the primer GS06.

\section{Authors' contributions}

JVLL carried out the molecular genetic studies, the obtention of the sequences, participated in the sequence alignment and analysis and drafted the manuscript. JBC carried out the phylogenetic and sequence analysis and drafted the manuscript. IMG participated in the sequence alignment. SS participated in the submission of the sequences to the EMBL Nucleotide Sequence Database and the draft the manuscript. RF participated in the conception and the design of the study. MJMS conceived and designed the study and coordinated and helped to draft the manuscript. All authors read and approved the final manuscript.

\section{Acknowledgements}

This work was supported by a grant (BMC2000-1446) from the Spanish Ministerio de Ciencia y Tecnología $(I+D)$.

\section{References}

I. Kim A, Terzian C, Santamaria P, Pelisson A, Purd'homme N, Bucheton $A$ : Retroviruses in invertebrates: the gypsy retrotransposon is apparently an infectious retrovirus of Drosophila melanogaster. Proceedings of the National Academy of Sciences of the United States of America 1994, 9 I (4): I 285- 289.

2. Pélisson A, Song SU, Prud'homme N, Smith PA, Bucheton A, Corces VG: Gypsy transposition correlates with the production of a retroviral envelope-like protein under the tissue-specific control of the Drosophila flamenco gene. The EMBO journal 1994, I3( I 8):440 I-44II.

3. Pélisson A, Teysset L, Chalvet F, Kim A, Prud'homme N, Terzian C, Bucheton A: About the origin of retroviruses and the co-evolution of the gypsy retrovirus with the Drosophila flamenco host gene. Genetica 1997, 100(I-3):29-37.

4. Mejlumian L, Pelisson A, Bucheton A, Terzian C: Comparative and functional studies of Drosophila species invasion by the gypsy endogenous retrovirus. Genetics 2002, 160(I):20I-209.

5. de Frutos R, Peterson KR, Kidwell MG: Distribution of Drosophila melanogaster transposable element sequences in species of the obscura group. Chromosoma 1992, 10 I(5-6):293-300.

6. Terzian C, Ferraz C, Demaille J, Bucheton A: Evolution of the Gypsy endogenous retrovirus in the Drosophila melanogaster subgroup. Mol Biol Evol 2000, I7(6):908-9|4.

7. Vázquez-Manrique RP, Hernández M, Martínez-Sebastián MJ, de Frutos R: Evolution of gypsy endogenous retrovirus in the Drosophila obscura species group. Mol Biol Evol 2000, I7(8): II85-1193.

8. Martínez-Sebastián MJ, Hernández M, Mejias B, Gas ME, Pérez A, Pascual L, de Frutos R: Evolutionary patterns of the gypsy and bilbo retrotransposon families in the Drosophila species of the obscura group. Molecular phylogenetics and evolution 2002, 25(2):254-266.

9. Heredia F, Loreto EL, Valente VL: Complex evolution of gypsy in Drosophilid species. Molecular biology and evolution 2004, 21(10): $1831-1842$.

10. Alberola TM, de Frutos R: Molecular structure of a gypsy element of Drosophila subobscura (gypsyDs) constituting a degenerate form of insect retroviruses. Nucleic acids research 1996, 24(5):914-923.

II. Flavell AJ: Long terminal repeat retrotransposons jump between species. Proceedings of the National Academy of Sciences of the United States of America 1999, 96(22): 1221 I-I22I2.

12. de Almeida LM, Carareto CM: Multiple events of horizontal transfer of the Minos transposable element between Drosophila species. Molecular phylogenetics and evolution 2005, 35(3):583-594.

13. Kotnova AP, Glukhov IA, Karpova NN, Salenko VB, Lyubomirskaya $\mathrm{NV}$, llyin YV: Evidence for recent horizontal transfer of gypsyhomologous LTR-retrotransposon gtwin into Drosophila erecta followed by its amplification with multiple aberrations. Gene 2007, 396(I):39-45.

14. Sánchez-Gracia A, Maside X, Charlesworth B: High rate of horizontal transfer of transposable elements in Drosophila. Trends Genet 2005, 2 I (4):200-203.

15. Silva JC, Loreto EL, Clark JB: Factors that affect the horizontal transfer of transposable elements. Current issues in molecular biology 2004, 6(I):57-7I.

16. Kidwell MG: Lateral transfer in natural populations of eukaryotes. Annual review of genetics 1993, 27:235-256.

17. Clark JB, Kidwell MG: A phylogenetic perspective on $\mathbf{P}$ transposable element evolution in Drosophila. Proceedings of the 
National Academy of Sciences of the United States of America 1997, 94(2I): I |428-I I 433.

18. García-Planells J, Paricio N, Clark JB, de Frutos R, Kidwell MG: Molecular evolution of $P$ transposable elements in the Genus drosophila. II. The obscura species group. Journal of molecular evolution 1998, 47(3):282-291.

19. Mizrokhi LJ, Mazo AM: Cloning and analysis of the mobile element gypsy from D. virilis. Nucleic acids research 1991, 19(4):913-916.

20. Barrio E, Ayala FJ: Evolution of the Drosophila obscura species group inferred from the Gpdh and Sod genes. Molecular phylogenetics and evolution 1997, 7(I):79-93.

21. O'Grady PM: Reevaluation of phylogeny in the Drosophila obscura species group based on combined analysis of nucleotide sequences. Molecular phylogenetics and evolution 1999, I2(2): 124-139.

22. O'Grady PM, Kidwell MG: Phylogeny of the subgenus sophophora (Diptera: drosophilidae) based on combined analysis of nuclear and mitochondrial sequences. Molecular phylogenetics and evolution 2002, 22(3):442-453.

23. Terzian C, Pelisson A, Bucheton A: Evolution and phylogeny of insect endogenous retroviruses. BMC evolutionary biology 200I, I:3.

24. Prud'homme N, Gans M, Masson M, Terzian C, Bucheton A: Flamenco, a gene controlling the gypsy retrovirus of Drosophila melanogaster. Genetics 1995, I39(2):697-7II.

25. Akashi H: Synonymous codon usage in Drosophila melanogaster: natural selection and translational accuracy. Genetics 1994, I36(3):927-935.

26. Ludwig A, Valente VL, Loreto EL: Multiple invasions of Errantivirus in the genus Drosophila. Insect molecular biology 2008, I7(2): II3-124.

27. Kosakovsky Pond SL, Posada D, Gravenor MB, Woelk CH, Frost SD: Automated phylogenetic detection of recombination using a genetic algorithm. Molecular biology and evolution 2006, 23(10): $1891-1901$.

28. Tamura K, Dudley J, Nei M, Kumar S: MEGA4: Molecular Evolutionary Genetics Analysis (MEGA) software version 4.0. Molecular biology and evolution 2007, 24(8): 1596-1599.

29. Nei M, Gojobori T: Simple methods for estimating the numbers of synonymous and nonsynonymous nucleotide substitutions. Mol Biol Evol 1986, 3(5):4I 8-426.

Publish with Bio Med Central and every scientist can read your work free of charge

"BioMed Central will be the most significant development for disseminating the results of biomedical research in our lifetime. "

Sir Paul Nurse, Cancer Research UK

Your research papers will be:

- available free of charge to the entire biomedical community

- peer reviewed and published immediately upon acceptance

- cited in PubMed and archived on PubMed Central

- yours - you keep the copyright
BioMedcentral 